\title{
Computer-based video analysis identifies infants with absence of fidgety movements
}

\author{
Ragnhild Støen ${ }^{1}$, Nils Thomas Songstad ${ }^{2}$, Inger Elisabeth Silberg ${ }^{3}$, Toril Fjørtoft ${ }^{1}$, Alexander Refsum Jensenius ${ }^{4}$, Lars Adde ${ }^{1}$ \\ on behalf of the CIMA Norway Study Group ${ }^{5}$
}

BACKGROUND: Absence of fidgety movements (FMs) at 3 months' corrected age is a strong predictor of cerebral palsy (CP) in high-risk infants. This study evaluates the association between computer-based video analysis and the temporal organization of FMs assessed with the General Movement Assessment (GMA).

METHODS: Infants were eligible for this prospective cohort study if referred to a high-risk follow-up program in a participating hospital. Video recordings taken at 10-15 weeks post term age were used for GMA and computer-based analysis. The variation of the spatial center of motion, derived from differences between subsequent video frames, was used for quantitative analysis.

RESULTS: Of 241 recordings from 150 infants, 48 (24.1\%) were classified with absence of FMs or sporadic FMs using the GMA. The variation of the spatial center of motion $\left(C_{S D}\right)$ during a recording was significantly lower in infants with normal (0.320; 95\% confidence interval (Cl) 0.309, 0.330) vs. absence of or sporadic $(0.380 ; 95 \% \mathrm{Cl} 0.361,0.398) \mathrm{FMs}(P<0.001)$. A triage model with $C_{S D}$ thresholds chosen for sensitivity of $90 \%$ and specificity of $80 \%$ gave a $40 \%$ referral rate for GMA.

CONCLUSION: Quantitative video analysis during the FMs' period can be used to triage infants at high risk of CP to early intervention or observational GMA.

E arly identification of infants at high risk of CP is essential in clinical practice to select an appropriate follow-up, and in research to develop and evaluate early interventions. Despite well-known risk factors for CP like preterm birth and perinatal asphyxia, a majority of those infants will have normal motor development (1). Less than $15 \%$ of infants born before 28 weeks of gestation develop CP (2), and, among extremely preterm infants with major brain injury identified on cerebral ultrasound, only a third develop CP (3). Lack of accurate and early markers for CP hampers the organization of resource-effective follow-up programs and research on early, high-intensity training programs.

Prognostic models based on gestational age, neonatal morbidities, and therapeutic interventions like the use of steroids in the neonatal period (4), as well as neurological assessment tools $(5,6)$, magnetic resonance imaging (7), or a combination of these $(8,9)$, may be used to predict outcomes in high-risk infants. A common finding is that accuracy of neurological assessment increases with age on assessment, delaying interventions beyond the time when brain plasticity is assumed to be at its highest $(10,11)$. The General Movement Assessment (GMA) has been found to have the best accuracy and predictive power for CP in young infants (12). The absence of so-called fidgety movements (FMs) at 3 months post term age has been shown to identify infants with CP with sensitivities between 90 and $98 \%$ and specificities between 90 and 94\% (refs 12-14). Recently, GMA was recommended as a screening tool for CP in high-risk infants in Australia (13).

The GMA requires ample training, frequent recalibration of analytical skills, and time for video observation and analysis. The GMA is based on Gestalt perception, and similar to other observational pattern-recognition methods for analysis of complex phenomena, it is in its nature subjective. Although inter-rater reliability appears high for experienced observers $(15,16)$, results are inconsistent (17). There will be a high demand for skilled observers if GMA is to be used in largescale screening. Because of these limitations, several automated, computer-based methods for pattern recognition of infants' spontaneous movements are under development (18). We have previously published results from a feasibility study of a computer-based video analysis software for quantitative analysis of infants' general movements (GMs) $(19,20)$. In a convenience sample of high-risk infants, the software identified infants with FMs with a sensitivity and specificity of $81.5 \%$ and $85 \%$, respectively. On the basis of these findings, we wanted to validate the method in a prospectively collected sample of high-risk infants who were routinely enrolled in a high-risk follow-up program at discharge from the neonatal intensive care unit.

${ }^{1}$ St. Olavs Hospital, Trondheim University Hospital, Faculty of Medicine, Norwegian University of Science and Technology, Norway; ${ }^{2}$ Department of Pediatrics and Adolescen Medicine, University Hospital of North Norway, Tromsø, Norway; ${ }^{3}$ Division of Paediatric and Adolescent Medicine, Department of Pediatrics, Oslo University Hospital, Oslo, Norway; ${ }^{4}$ Department of Musicology, University of Oslo, Oslo, Norway. Correspondence: Ragnhild Støen (ragnhild.stoen@ntnu.no)

${ }^{5}$ Members of the CIMA Norway study group are listed above References.

Received 11 December 2016; accepted 7 May 2017; advance online publication 26 July 2017. doi:10.1038/pr.2017.121 


\section{Articles | Stoen et al.}

The aim of the present study was to explore whether selected computer-based movement variables were associated with the temporal organization of FMs in infants at risk of perinatal brain injury. Furthermore, we wanted to explore a risk stratification model based on thresholding of computerbased movement variables to differentiate between infants with normal vs. absence of or sporadic FMs.

\section{METHODS}

\section{Participants}

A total of 165 infants born between 1 October 2008 and 31 December 2012 and designated to one of the four participating Norwegian hospitals' follow-up clinics at discharge from the neonatal intensive care unit were included. All infants were born and had their initial treatment in one of the three tertiary centers enrolling patients in the study. Infants who did not have their follow-up in a participating center, or infants with peripheral neuromotor diseases, e.g., brachial plexus injury, or iatrogenic restricted movements (e.g., cast for congenital talipes equinovarus), were not included in the study. Twelve infants were excluded because of errors in the standardized camera setup $(n=10)$ or crying during recording $(n=2)$. Three infants (all with $\mathrm{GA}<28$ weeks) were classified with exaggerated FMs. They were excluded from further analysis because their FMs were not classified according to their temporal organization. Table 1 shows the reason for referral to follow-up among the remaining 150 infants. Although one infant could have several risk factors for adverse development, all infants were classified into one risk group according to their main reason for referral. Extremely preterm infants $(\mathrm{GA}<28$ weeks and/or $\mathrm{BW} \leq 1,000 \mathrm{~g})$ were classified as such irrespective of other risk factors. Among the eight infants exposed to perinatal asphyxia who were not treated with therapeutic hypothermia (TH), three had a GA below 36 weeks and were not considered eligible for $\mathrm{TH}$. The remaining five infants were term infants, of whom one had an assumed prenatal insult, three had hypoxic-ischemic encephalopathy (HIE) stages I-II, and were not considered to fulfill the neurological criteria for $\mathrm{TH}$, and one infant had HIE stage II, which was not recognized until after $6 \mathrm{~h}$ of life.

\section{Video Recording}

Parents were invited to bring their infant for video recording once or twice (mainly depending on travel distance) between 10 and 15 weeks post term age. All recordings were performed during active wakefulness using a standardized setup with a commercially available digital video camera (Sanyo VPC-HD2000, SANYO Electric Co., Ltd., Osaka, Japan) on a tripod with a standardized distance and angle to the mattress where the infant was placed $(18,20)$. All infants were dressed in a white "onesie". Gestalt assessment of GMs and the

Table 1. Demographic variables and risk groups

\begin{tabular}{lc}
\hline Risk group & Individuals, $N=150$ \\
\hline BW $\leq 1$ kg and/or GA $<28$ weeks, $n(\%)$ & $72(48.0)$ \\
Boys, $n(\%)$ & $42(58.3)$ \\
GA (weeks), median (range) & $26.9(24.0-32.1)$ \\
BW (g), median (range) & $845(385-1,190)$ \\
NAIS, $n$ (\%) & $9(6.0)$ \\
Perinatal asphyxia and TH, $n(\%)$ & $32(21.3)$ \\
Perinatal asphyxia without TH, $n(\%)$ & $8(5.3)$ \\
Others ${ }^{\text {a }}, n$ (\%) & $29(19.3)$ \\
\hline BW, birth weight; CNS, central nervous system; GA, gestational age; NAIS, neonatal \\
arterial ischemic stroke; TH, therapeutic hypothermia. \\
aOthers: severe neonatal sepsis with multiorgan failure, CNS infection, twin-to-twin \\
transfusion syndrome, imaging abnormalities, neonatal seizures, growth restriction, \\
suspected or confirmed syndrome, and severe neonatal hypoglycemia.
\end{tabular}

computer-based analysis were based on video recordings performed according to the GMA methodology of Prechtl and co-workers (21). Thirty-eight $(13.6 \%)$ of 279 recordings, all from infants with two recordings, were excluded because of technical problems $(n=21)$, crying $(n=9)$, displacement of the infant on the mattress $(n=6)$, or unknown factors $(n=2)$. Ninety-one $(61 \%)$ of the included infants had two recordings, and fifty-nine (39\%) infants had one recording available for analysis. A total of 241 recordings from 150 infants were analyzed with both GMA and computer-based video analysis. The median post-menstrual age at recording was 52.0 (range 49-57) weeks. The median length of recording was 300 s (range 50-343).

\section{Observation of GMs}

Two certified observers, who had successfully completed the GMs Trust training courses, classified the GMs. Both observers were blinded to the medical history of the infants and observed the videos independently. In case of disagreement, the observers re-assessed the video together and reached consensus. FMs are small movements of moderate speed and variable acceleration, of neck, trunk, and limbs, in all directions (21), and their temporal organization was classified according to how frequently they occurred and the duration of interspersed pauses. FMs were defined as normal if present intermittently or continually. Continual FMs were interspersed with only short pauses, whereas intermittent FMs were interspersed with longer pauses, giving the impression that FMs were present for only half the recording time. Sporadic FMs were interspersed with even longer pauses. Exaggerated FMs were defined as presence of FMs, but excessive in amplitude and speed (21).

\section{Computer-Based Video Analysis of GMs}

The video analysis software has been described in detail previously $(19,20)$. The video contains 25 frames per second with a resolution of $1,280 \times 720$ pixels. By subtracting subsequent frames in the video stream (frame differencing), the pixels changing between frames are identified and used to create a "motion image". A motion image thus represents the motion happening between two consecutive frames in the video stream (22), and can further be used to extract quantitative data features. A motion image built from a matrix containing only zeros indicates that no movement occurs between the frames, whereas a motion image from a matrix with positive values represents movement. All videos in the present study were cropped to remove any non-infant movement (e.g., parents interfering with the infant), and so that only a window containing the mattress with the infant was available for analysis.

The quantitative variables were based on variables that have been shown previously to differ between infants with presence and absence of FMs (20). Quantity of motion $(Q)$ is calculated for each frame as the sum of all pixel values (active pixels indicating movement) in the motion image divided by the total number of pixels, to create a resolution-independent feature. The centroid of motion $(C)$ is the spatial center of the pixels with positive values in the motion image. This variable reflects the center point of the overall movement of the infant, and it will continuously change position during a video sequence. The SD of the centroid of motion $\left(C_{\mathrm{SD}}\right)$ was calculated as the vector magnitude of the $x$ (horizontal) and $y$ (vertical) directions of the spatial center of motion. Evenly distributed movements in all body parts in all directions will be the source of low variability of the $C_{\mathrm{SD}}$, regardless of large or small movement amplitudes. Infant movements characterized by unsteady limb movements (i.e., uneven upper and lower limb activity with respect to laterality) will typically have higher $C_{\mathrm{SD}}$ values.

\section{Statistical Analysis}

Data were analyzed using SPSS Statistics version 21.0 (IBM SPSS Statistics, Chicago, IL). To correct for variable body size between infants, all computer-based movement variables were normalized for the trunk area, which was calculated by multiplying the trunk length with the trunk width measured from the video image $\left(\mathrm{cm}^{2}\right)$. 


\section{Quantitative analysis of infant movement Articles}

Table 2. Temporal organization of FMs according to risk group for all recordings

\begin{tabular}{|c|c|c|c|c|}
\hline Temporal organization of FMs & Absent, $N(\%)$ & Sporadic, $N(\%)$ & Intermittent, $N(\%)$ & Continual, $N(\%)$ \\
\hline \multicolumn{5}{|l|}{ Risk group } \\
\hline $\mathrm{BW} \leq 1 \mathrm{~kg}$ and/or $\mathrm{GA}<28$ weeks & $10(9.1)$ & $9(8.2)$ & $83(75.5)$ & $8(7.3)$ \\
\hline NAIS & $5(33.3)$ & $1(6.7)$ & $8(53.3)$ & $1(6.7)$ \\
\hline Perinatal asphyxia and $\mathrm{TH}$ & $3(6.0)$ & $4(8.0)$ & $34(68.0)$ & $9(18.0)$ \\
\hline \multicolumn{5}{|l|}{ Perinatal asphyxia without } \\
\hline $\mathrm{TH}$ & $6(42.9)$ & 0 & $7(50.0)$ & $1(7.1)$ \\
\hline Others $^{a}$ & $16(30.8)$ & $4(7.7)$ & $25(48.1)$ & $7(13.5)$ \\
\hline Total & $40(16.6)$ & $18(7.5)$ & $157(65.1)$ & $26(10.8)$ \\
\hline
\end{tabular}

BW, birth weight; CNS, central nervous system; FM, fidgety movement; GA, gestational age; NAIS, neonatal arterial ischemic stroke; TH, therapeutic hypothermia.

${ }^{a}$ Others: severe neonatal sepsis with multiorgan failure, CNS infection, twin-to-twin transfusion syndrome, imaging abnormalities, neonatal seizures, growth restriction, suspected or confirmed syndrome, and severe neonatal hypoglycemia.

The computer-based movement variables $C_{\mathrm{SD}}$ and $Q$ were compared between the groups of FMs' temporal organization using a linear mixed model. A subject-specific, random intercept was included to account for a possible within-subject correlation for the individuals with two repeated measurements. Temporal organization of FM was included as a categorical covariate, and gestational age as a continuous covariate.

To study the predictive abilities of the $C_{\mathrm{SD}}$ for the binary outcome of normal vs. absence of or sporadic FMs, a generalized linear mixed model was applied using $C_{\mathrm{SD}}$ as a covariate. The area under the curve was used to assess the discrimination between normal and absence of or sporadic FMs based on the generalized linear mixed model. A triage model comprising the three groups "refer to early intervention", "refer to GMA", and "reassure" was selected based on combinations of sensitivities and specificities.

Results were considered statistically significant for $P$ values less than 0.05 .

\section{Ethical Approval}

The study was approved by the regional committee for medical and health research ethics (REC Central-Committee 4.2007.2327). Written parental consent was obtained before inclusion.

\section{RESULTS}

Table 2 shows the temporal organization of FMs according to clinical risk group for all recordings. The highest proportion of absence of and sporadic FMs was found in recordings from infants diagnosed with neonatal arterial ischemic stroke and those with perinatal asphyxia not treated with $\mathrm{TH}$.

The $C_{\mathrm{SD}}$ was significantly lower in infants with normal $(0.320 ; 95 \%$ confidence interval (CI) $0.309,0.330)$ vs. those with absence of or sporadic $(0.380 ; 95 \%$ CI $0.361,0.398) \mathrm{FMs}$ $(P<0.001)$. There was a significant increase in $C_{\mathrm{SD}}$ from continual to intermittent to absence of or sporadic FMs (Figure 1a). No significant overall difference in $Q$ was found between different categories of temporal organization of FMs (Figure 1b). Adjusting for gestational age did not affect any of the results.

The area under curve for the $C_{\mathrm{SD}}$ was 0.73 (95\% CI 0.658 , 0.800 ) for normal (intermittent or continual) vs. absence of FMs or sporadic FMs using $C_{\mathrm{SD}}$ as the only covariate (generalized linear mixed model). Different triage models were explored. Choosing a model with sensitivity and specificity of $80 \%$ resulted in a $27 \%$ referral rate to GMA. With this model, there was a false-negative rate for the computer-based video analysis (a $C_{\mathrm{SD}}$ value below the lower threshold despite absence of or sporadic FMs assessed with GMA) of $20 \%$. Increasing the sensitivity to $90 \%$ resulted in a referral rate to GMA of $40 \%$ and a false-negative rate of $10 \%$ (Table 3). With both models, $50 \%$ of recordings above the upper threshold (refer to early intervention) were assessed with absence of or sporadic FMs using GMA.

\section{DISCUSSION}

This study confirms that computer-based video analysis applied on standardized recordings of GMs in young infants can provide objective information about the presence or absence of normal FMs. A larger variability of the spatial center of movements throughout a video was strongly associated with the absence of normal FMs, and thereby is a high risk for later CP. The lowest variation of the spatial center of movement was seen in infants with continual presence of FMs. This automated movement analysis can easily be applied for screening of high-risk infants to select those who need referral to an observational GMA.

Eligible infants in the present study were those who are routinely enrolled in follow-up clinics because of increased risk of adverse neurodevelopment. Criteria for such follow-up will differ between hospitals, but commonly comprise extremely preterm infants as well as more mature infants with perinatal morbidities known to increase the risk of perinatal brain injury. Associations between Gestalt perception and computer-based analysis were consistent across the different risk groups and were not affected by gestational age. Consequently, the included sample mirrors the diversity seen in the group of infants who are at risk of perinatal brain injury, and the results are not restricted to infants with specific underlying brain pathology.

Increased frequency of FMs in a video recording, from absence or sporadic to intermittent to continual, was strongly associated with a decrease in the variability of the spatial 

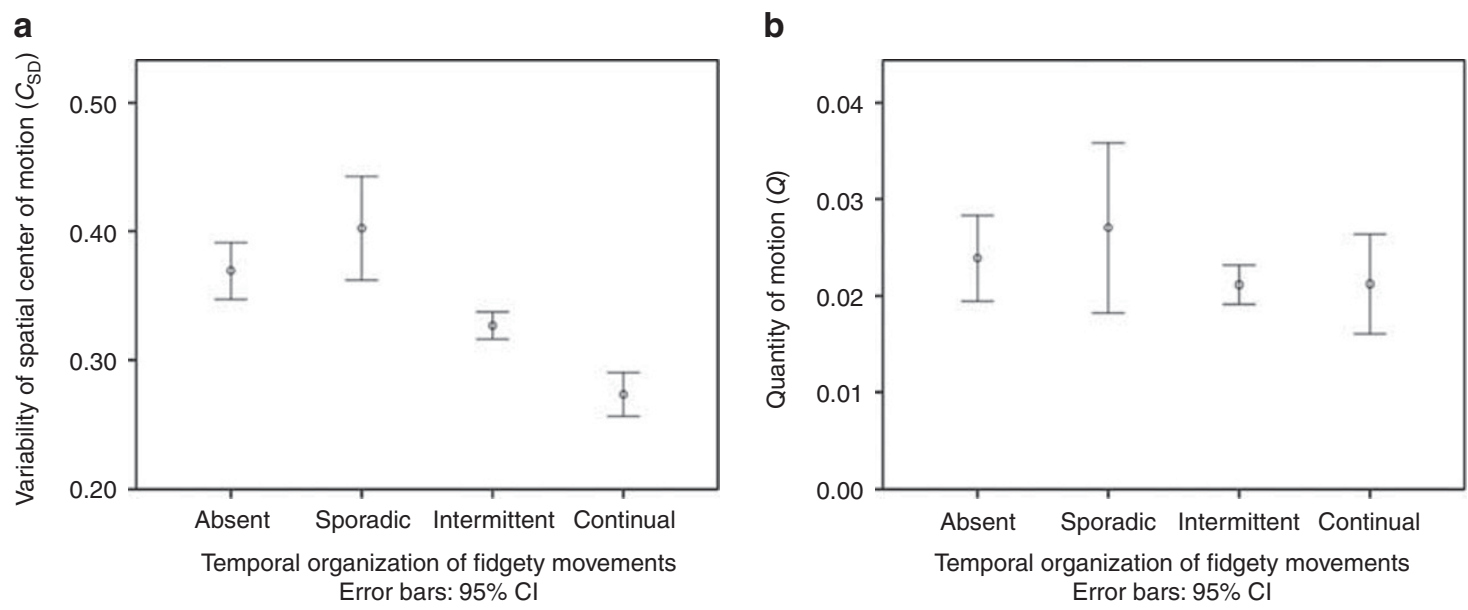

Figure 1. Computer-based movement variables and temporal organization of fidgety movements. (a) Centroid of motion $\left(C_{S \mathrm{D}}\right)$ according to temporal organization of fidgety movements (FMs) in all recordings. Statistically significant difference between absence of and sporadic FMs vs. intermittent and continual FMs with $P<0.001$. (b) Quantity of motion $(Q)$ according to temporal organization of FMs in all recordings. No significant differences between groups. $\mathrm{Cl}$, confidence interval.

Table 3. Triage threshold analysis of variability of $C_{\mathrm{SD}}$

\begin{tabular}{lcc}
\hline Threshold $C_{\mathrm{SD}}$ & $\begin{array}{c}\text { Sensitivity } 80 \% ; \\
\text { specificity } 80 \%\end{array}$ & $\begin{array}{c}\text { Sensitivity } 90 \% ; \\
\text { specificity } 80 \%\end{array}$ \\
\hline Upper threshold: & 0.377521 & 0.427189 \\
Lower threshold: & 0.312323 & 0.312323 \\
& $N(\%)$ & $N(\%)$ \\
\hline
\end{tabular}

Above upper threshold: refer to early intervention

$\begin{array}{lll}\text { Absence of or sporadic FMs } & 30(50.8) & 30(50.8) \\ \text { Normal FMs } & 36(19.8) & 36(19.8)\end{array}$

Between thresholds: refer to GMA

Absence of or sporadic FMs

$17(28.8)$

$23(39.0)$

Normal FMs

$49(26.9)$

$74(40.7)$

Below lower threshold: reassure

Absence of or sporadic FMs

$12(20.3)$

$6(10.2)$

Normal FMs

$97(53.3)$

$72(39.6)$

Referral rate to GMA

$27 \%$

$40 \%$

$C_{S D}$, SD of the centroid of motion; FM, fidgety movement; GMA, General Movement Assessment.

center of motion. This association between the temporal organization of FMs and the $C_{\mathrm{SD}}$ has also been found in healthy, term-born infants (23). An explanation may be that FMs, which involve the whole body in an evenly distributed way, lead to a more stable center of motion. During periods with no FMs in a video, the variability of the center of motion will be the result of more unevenly distributed limb and body movements, such as isolated limb movements, asymmetric posture, and potentially stiff, jerky, or monotonous movements. The quantity of motion $(Q)$ did not increase significantly with increasing presence of FMs in the video.
This variable most likely measures a combination of FMs and concurrent movements, and will not differentiate between healthy and abnormal concurrent movements.

Several methods for automated movement analysis have shown discriminative power with regard to later CP or other developmental delays in small groups of high-risk infants (24-26). Using magnetic sensors, Philippi et al. (26) demonstrated that stereotypic arm movements predicted CP with high accuracy in a group of 49 infants. Kanemaru et al. (25), using reflective tape as limb sensors, found that children born preterm who exhibited developmental delay at 3 years of age had less active spontaneous movements at term-equivalent age. The video analysis in the present study is based on a sensor-less system to increase clinical feasibility. The movement variables extracted from the videos are not limited to one or more limbs, but reflect a more holistic measure of movement patterns. We suggest that this is more in accordance with the Gestalt perception used in GMA (27). However, adding features like frequency of limb movements (separately or in combination) may add to the accuracy of our method and needs to be further explored using more advanced computer vision methods (28). It also remains to be systematically tested as to how changes in camera distance or angle, or different infant clothing, influence the robustness of the computer-based method. This is particularly important if a future goal is to facilitate the implementation of computer-based methods in smart phone applications developed for remote GMA.

The purpose of early identification of CP risk is to refer infants to high-intensity intervention, highlighting the importance of high sensitivity of the test. However, avoidance of unnecessary follow-up and reassurance to parents of a healthy motor development will also have high priority for those working with children at risk. In the present study, a sensitivity of $80 \%$ had a corresponding specificity of $53 \%$, meaning that a rather large proportion of infants with normal 


\section{\begin{tabular}{l|l} 
Quantitative analysis of infant movement & Articles
\end{tabular}}

FMs would be regarded as potentially abnormal based on the automated video analysis. A triage model, where those between upper and lower threshold are referred for an observational GMA, is suggested to provide more certainty to parents and reduce unnecessary worries. The accuracy of early identification may also be improved by combining computerbased analysis with other assessments like neurological examination or magnetic resonance imaging, depending on the clinical setting and availability of resources.

Both observers who classified GMA in the present study have completed two advanced GMA courses each and have more than 15 years' experience using GMA in clinical practice and research. Predictive accuracy of GMA during the FMs' period for later CP has been reported to be very high $(13,27)$, and inter-rater reliability appears to be high $(15,16)$. A recent report from Australia showed very high sensitivity and specificity for abnormal development at 1 year when GMA was assessed by several observers in a structured GMA network (13). That study, however, used only three categories of FMs, i.e., absent, present, or abnormal (exaggerated). To our knowledge, inter-rater reliability of temporal organization of FMs using all available categories has not been published. The definition of sporadic FMs as lasting 1-3 s with long interspersed pauses (up to $1 \mathrm{~min}$ ) came recently (29), and the significance of sporadic FMs for later CP development is not entirely clear. Quantitative movement analysis of infants during the FMs' period comprising all categories of temporal organization may contribute to increased knowledge and understanding of FMs as a phenomenon.

\section{Interpretation}

This study confirms that automated movement analysis captures movement features relevant for the classification of FMs in high-risk infants. A low variability of the spatial center of motion throughout a video was strongly associated with normal FMs. A triage model based on automated movement analysis reduced the need for observational GMA, and half of the infants with absence of or sporadic FMs could be referred to early intervention based on automated movement analysis alone. Further studies including long-term outcomes are needed to explore the use of automated movement analysis as an adjunct to observational GMA for prediction of $\mathrm{CP}$ in infants at risk of perinatal brain injury.

\section{ACKNOWLEDGMENTS}

We want to thank associate professor of medical statistics, Turid Follestad, at the Faculty of Medicine, NTNU, for help with statistical analysis.

\section{STATEMENT OF FINANCIAL SUPPORT}

This study was supported by grant from The Liaison Committee between the Central Norway Regional Health Authority (RHA) and the Norwegian University of Science and Technology (NTNU) and the Joint Research Committee between St. Olavs Hospital and the Faculty of Medicine, NTNU.

\section{DISCLAIMER}

The funder was not involved in study design, data collection, analysis, or preparation of the manuscript.
ADDITIONAL MEMBERS OF THE CIMA NORWAY STUDY GROUP

Cathrine Labori ${ }^{7}$, Marianne Loennecken ${ }^{8}$, Unn Inger Möinichen ${ }^{8}$, Randi Tynes Vaagen ${ }^{9,10}$, Gunn Kristin Øberg ${ }^{11,12}$

${ }^{7}$ Department of Clinical Therapeutic Services, University Hospital of North Norway, Tromsø, Norway; ${ }^{8}$ Department of Pediatrics, Division of Paediatric and Adolescent Medicine, Oslo University Hospital, Oslo, Norway; ${ }^{9}$ Clinics of Clinical Services, St. Olavs Hospital, Trondheim University Hospital, Norway; ${ }^{10}$ Department of Laboratory Medicine, Children's and Women's Health, Norwegian University of Science and Technology, Trondheim, Norway; ${ }^{11}$ Department of Health and Care Sciences, Faculty of Health Sciences, University of Tromsø, The Arctic University of Norway, Troms $\varnothing$, Norway; ${ }^{12}$ Department of Clinical Therapeutic Services, University Hospital of North Norway, Tromsø, Norway.

Disclosure: The authors declare no conflict of interest.

\section{REFERENCES}

1. Herskind A, Greisen G, Nielsen JB. Early identification and intervention in cerebral palsy. Dev Med Child Neurol 2015;57:29-36.

2. Himpens E, Van den Broeck C, Oostra A, Calders P, Vanhaesebrouck P. Prevalence, type, distribution, and severity of cerebral palsy in relation to gestational age: a meta-analytic review. Dev Med Child Neurol 2008;50: $334-40$.

3. Bolisetty S, Dhawan A, Abdel-Latif M, Bajuk B, Stack J, Lui K. Intraventricular hemorrhage and neurodevelopmental outcomes in extreme preterm infants. Pediatrics 2014;133:55-62.

4. Linsell L, Malouf R, Morris J, Kurinczuk JJ, Marlow N. Prognostic factors for cerebral palsy and motor impairment in children born very preterm or very low birthweight: a systematic review. Dev Med Child Neurol 2016;58:554-69.

5. Lekskulchai R, Cole J. Effect of a developmental program on motor performance in infants born preterm. Aust J Physiother 2001;47:169-76.

6. Noble Y, Boyd R. Neonatal assessments for the preterm infant up to 4 months corrected age: a systematic review. Dev Med Child Neurol 2012;54:129-39.

7. Anderson PJ, Cheong JL, Thompson DK. The predictive validity of neonatal MRI for neurodevelopmental outcome in very preterm children. Semin Perinatol 2015;39:147-58.

8. Setanen S, Lahti K, Lehtonen L, et al. Neurological examination combined with brain MRI or cranial US improves prediction of neurological outcome in preterm infants. Early Hum Dev 2014;90:851-6.

9. Skiold B, Eriksson C, Eliasson AC, Aden U, Vollmer B. General movements and magnetic resonance imaging in the prediction of neuromotor outcome in children born extremely preterm. Early Hum Dev 2013;89:467-72.

10. Fiori S, Guzzetta A. Plasticity following early-life brain injury: insights from quantitative MRI. Semin Perinatol 2015;39:141-6.

11. Staudt M. Reorganization after pre- and perinatal brain lesions. J Anat 2010;217:469-74.

12. Bosanquet M, Copeland L, Ware R, Boyd R. A systematic review of tests to predict cerebral palsy in young children. Dev Med Child Neurol 2013;55:418-26.

13. Morgan C, Crowle C, Goyen TA, et al. Sensitivity and specificity of general movements assessment for diagnostic accuracy of detecting cerebral palsy early in an Australian context. J Paediatr Child Health 2016;52:54-9.

14. Oberg GK, Jacobsen BK, Jorgensen L. Predictive value of General Movement Assessment for cerebral palsy in routine clinical practice. Phys Ther 2015;95:1489-95.

15. Fjortoft T, Einspieler C, Adde L, Strand LI. Inter-observer reliability of the "assessment of motor repertoire-3 to 5 months" based on video recordings of infants. Early Hum Dev 2009;85:297-302.

16. Valentin T, Uhl K, Einspieler C. The effectiveness of training in Prechtl's method on the qualitative assessment of general movements. Early Hum Dev 2005;81:623-7.

17. Bernhardt I, Marbacher M, Hilfiker R, Radlinger L. Inter- and intraobserver agreement of Prechtl's method on the qualitative assessment of 


\section{Articles | stoen et al.}

general movements in preterm, term and young infants. Early Hum Dev 2011;87:633-9.

18. Marcroft C, Khan A, Embleton ND, Trenell M, Plotz T. Movement recognition technology as a method of assessing spontaneous general movements in high risk infants. Front Neurol 2014;5:284.

19. Adde L, Helbostad JL, Jensenius AR, Taraldsen G, Grunewaldt KH, Stoen R. Early prediction of cerebral palsy by computer-based video analysis of general movements: a feasibility study. Dev Med Child Neurol 2010;52:773-8.

20. Adde L, Helbostad JL, Jensenius AR, Taraldsen G, Stoen R. Using computer-based video analysis in the study of fidgety movements. Early Hum Dev 2009;85:541-7.

21. Einspieler C, Prechtl HFR, Bos AF, Ferrari FCG. Prechtl's Method on the Qualitative Assessment of General Movements in Preterm, Term and Young Infants. London, UK: Mac Keith Press, 2004.

22. Jensenius AR. Some video abstraction techniques for displaying body movement in analysis and performance. Leonardo 2013;46:53-60.

23. Valle SC, Stoen R, Saether R, Jensenius AR, Adde L. Test-retest reliability of computer-based video analysis of general movements in healthy termborn infants. Early Hum Dev 2015;91:555-8.
24. Heinze F, Hesels K, Breitbach-Faller N, Schmitz-Rode T, DisselhorstKlug C. Movement analysis by accelerometry of newborns and infants for the early detection of movement disorders due to infantile cerebral palsy. Med Biol Eng Comput 2010;48:765-2.

25. Kanemaru N, Watanabe H, Kihara $\mathrm{H}$, et al. Jerky spontaneous movements at term age in preterm infants who later developed cerebral palsy. Early Hum Dev 2014;90:387-92.

26. Philippi H, Karch D, Kang KS, et al. Computer-based analysis of general movements reveals stereotypies predicting cerebral palsy. Dev Med Child Neurol 2014;56:960-7.

27. Prechtl HF, Einspieler C, Cioni G, Bos AF, Ferrari F, Sontheimer D. An early marker for neurological deficits after perinatal brain lesions. Lancet 1997;349:1361-3.

28. Rahmati H, Martens H, Aamo OM, Stavdahl O, Stoen R, Adde L. Frequency analysis and feature reduction method for prediction of cerebral palsy in young infants. IEEE Trans Neural Syst Rehabil Eng 2016;24:1225-34.

29. Einspieler C, Yang H, Bartl-Pokorny KD, et al. Are sporadic fidgety movements as clinically relevant as is their absence? Early Hum Dev 2015;91:247-52. 